\title{
Alimentación y actividad física: un reto de nuestro tiempo
}

\author{
Diet and physical activity: A current challenge
}

\author{
Félix Lobo
}

Departamento de Economía, Universidad Carlos III, Madrid, España

En este número de Medicina Clínica se publica un importante trabajo que estima en el $11,2 \%$ la prevalencia de conductas de riesgo relacionadas con el miedo a ganar peso, el impulso de adelgazar y la presencia de patrones alimentarios restrictivos entre jóvenes españoles ${ }^{1}$. Estas conductas pueden conducir a trastornos de la conducta alimentaria, que ya constituyen la tercera enfermedad crónica más prevalente entre las adolescentes en los países desarrollados.

Este comentario editorial quiere encuadrar dicho trabajo en un marco más amplio y situarlo en un conjunto de problemas alimentarios y de inactividad física que va mucho más allá de los trastornos patológicos que requieren como remedio de atención psicológica o psiquiátrica personal, sino también de respuestas colectivas que pongan en cuestión una visión social del cuerpo humano que exalta, de forma desaforada, una imagen estereoti pada, que sólo valora la delgadez.

Efectivamente, en la sociedad española, como en casi todo el mundo, incluidos muchos países en vías de desarrollo o, incluso, claramente subdesarrollados, la obesidad se ha convertido ya en un problema de salud pública de primer orden. Las cifras y su evolución temporal son elocuentes. Cualquiera que sea el criterio de medida que utilicemos (índice de masa corporal, perímetro abdominal, tablas específicas para niños y adolescentes), las tendencias hacia el crecimiento del sobrepeso y la obesidad parecen imparables. También está claro, a partir de los datos, que hay un gradiente social de la obesidad. Los grupos sociales más favorecidos, en términos de renta y de educación, sufren menos el problema, o incluso en algunos países lo han afrontado y resuelto, mientras que los grupos menos favorecidos empeoran de forma acusada a lo largo del tiempo.
Todos sabemos que la obesidad no es sólo un problema estético. Están ya bien consolidadas algunas conclusiones acerca del potencial que tiene como generadora de morbilidad, mortalidad prematura, pérdida de calidad de vida y discapacidad. La propensión a sufrir algunas enfermedades crece con la obesidad. Este es el caso, como es bien sabido, de la diabetes tipo 2, de las enfermedades cardiovasculares y también de ciertas dolencias músculo esqueléticas y formas de cáncer. Especialmente preocu pante es el sobrepeso y la obesidad infantil, pues los niños o adolescentes con sobrepeso y obesidad hoy, serán enfermos mañana. Quizás el fenómeno que mejor exprese esta inquietante evolución es que la diabetes tipo 2 está dejando de ser una enfermedad característica de la madurez y la edad avanzada por su progresión en poblaciones cada vez más jóvenes.

Además de estos graves problemas de salud que la obesidad genera y que se traducen en una disminución de los años de vida y de su calidad, también produce problemas y costes socioeconó micos. Por un lado, por sí misma y por las enfermedades que desencadena o favorece, la obesidad genera importantes costes para los sistemas sanitarios. La Organización para el Cooperación y el Desarrollo Económico (OCDE) ha reiterado que la viabilidad futura de los sistemas sanitarios depende no sólo de mejoras en la financiación, la organización o la gestión, sino también de la contención o la disminución significativa de problemas de salud pública como el tabaquismo, el alcoholismo y la obesidad. Atajar estos problemas permitiría liberar una inmensa masa de recursos que podríamos dedicar a la atención de las enfermedades degenerativas y otras crónicas características de sociedades envejecidas, y a financiar innovaciones tecnológicas que, sin duda, resolverán problemas de salud hoy insolubles o que mejorarán nuestra calidad de vida en formas todavía inalcanzables.

El desarrollo de la investigación científica, en campos de especialización muy diversos, está empezando a desvelar las causas del crecimiento de la obesidad. Quizás la principal conclusión es su naturaleza multifactorial. Existen factores 
genéticos, fisiológicos específicos, psicológicos y también sociales y económicos. Otra conclusión es la interrelación entre alimen tación y actividad física, binomio inseparable impuesto por el balance fisiológico entre consumo y gasto de energía. Ambas dimensiones son importantes y ambas han de ser tenidas en cuenta en cualquier estrategia, individual o colectiva, de lucha contra la obesidad.

Sin embargo, la experiencia demuestra que la batalla contra la obesidad y los trastornos alimentarios, dada la complejidad de sus factores etiológicos, es complicada y con resultados sólo en el medio y largo plazo. Por ello exige, para librarla, numerosos esfuerzos: progresar en la investigación y comprensión del fenómeno; adoptar legislación y políticas de acción eficientes por los poderes públicos, proporcionar educación e información a los niños, jóvenes, padres, mujeres en el climaterio y muchos otros colectivos sociales sensibles; regular y alinear los incentivos de las empresas (especialmente de las industrias de alimentación, bebidas, hostelería, del ocio y ejercicio físico) para que encuentren beneficiosos los productos saludables y se comprometan con la salud; mejorar, en fin, las actuaciones del sistema sanitario en atención primaria y especializada en todas las fases de prevención, diagnóstico, tratamiento y seguimiento.

Los organismos internacionales y los gobiernos ya han comenzado a crear un cuerpo de doctrina y a desarrollar programas de acción para atajar la obesidad. La Organización Mundial de la Salud lanzó en 2004 su Estrategia Mundial sobre Régimen Alimentario, Actividad Física y Salud ${ }^{2}$ (aprobada por la resolución WHA57.17). Llevaba tiempo advirtiendo de la importancia creciente de las enfermedades no transmisibles y sus factores de riesgo principales: hipertensión arterial, hipercolesterolemia, escasa ingesta de frutas y hortalizas, exceso de peso u obesidad, falta de actividad física y consumo de tabaco. Como se dice en la Estrategia, cinco de estos factores de riesgo están estrechamente asociados a la mala alimentación y la falta de actividad física.

El documento básico de la Estrategia contiene datos, principios para gobernar políticas y medidas contra la obesidad y distribución de responsabilidades de actuación entre todos los organismos y agentes, incluido el sector privado. Con respecto a la dieta hace cinco recomendaciones, ya clásicas: lograr un equilibrio energético y un peso normal; limitar la ingesta energética procedente de las grasas, sustituir las grasas saturadas por grasas insaturadas y tratar de eliminar los ácidos grasos trans; aumentar el consumo de frutas y hortalizas, así como de legumbres, cereales integrales y frutos secos; limitar la ingesta de azúcares libres; limitar la ingesta de sal (sodio) de toda procedencia y consumir sal yodada.

Con respecto a la actividad física, se recomienda que las personas se mantengan suficientemente activas durante toda la vida y al menos 30 minutos de actividad regular de intensidad moderada con una frecuencia casi diaria.

La Comisión de la Unión Europea publicó en 2007 su conciso Libro blanco con una Estrategia para Europa sobre nutrición, sobrepeso y obesidad ${ }^{3}$, importante documento que reconoce que la obesidad ha crecido dramáticamente en nuestra Unión en los treinta últimos años, siendo especialmente preocupante la evolución en la infancia. Proclama cuatro principios de acción: ir a la raíz del problema considerando todos los factores de riesgo, no sólo el sobrepeso; necesidad de atacar el problema en todas las políticas (enfoque horizontal) y a todos los niveles de gobierno (enfoque vertical); colaboración del sector privado (por ejemplo la industria alimentaria) y del nivel local (por ejemplo las escuelas); realizar siempre seguimiento y evaluación de los programas. La coherencia de la política a desarrollar por las instituciones europeas se basará en seis pilares: información para los consumidores; conseguir que las opciones saludables estén disponibles para la población; promover la actividad física; especial atención a grupos prioritarios como la infancia; desarrollo de un cuerpo de conocimiento con pruebas científicas demos tradas; desarrollo de sistemas de seguimiento y evaluación.

El enfoque adoptado, que domina toda la estrategia europea, es de cooperación entre todos los actores y en especial entre sector público y privado. En el plano de los países la Comisión reconoce el protagonismo de los estados miembros y la importancia de las acciones locales. Para apoyarlas favorecerá la difusión de programas ejemplares (best practices).

En España, el Ministerio de Sanidad y la Agencia Española de Seguridad Alimentaria y Nutrición, su organismo especializado en la materia, lanzaron en 2005 la Estrategia por la Nutrición, Actividad Física contra la Obesidad y por la Salud (Estrategia NAOS $)^{4}$. Tiene como meta fundamental fomentar la alimentación saludable y la actividad física, para invertir la tendencia ascendente de la prevalencia de la obesidad y, con ello, reducir sustancial mente la morbilidad y la mortalidad atribuibles a las enfermedades crónicas. Más que dar protagonismo absoluto a las administra ciones públicas, pretende principalmente movilizar los más amplios apoyos políticos y sociales e iniciativas de todo tipo.

Los principios de actuación que propone son:

1. Información y educación, que se consideran necesarias pero unidas imprescindiblemente a cambios en el entorno social, económico y cultural (ambiente obesogénico).

2. Evitar intervenciones aisladas y actuar de forma integral en colegios, familias, centros de trabajo y a nivel local.

3. Las acciones deben dirigirse a todos los grupos de edad, pero enfatizando sobre niños y jóvenes.

4. Es imprescindible que las opciones saludables sean accesibles a toda la población.

5. Necesidad de medir y evaluar periódicamente las acciones desarrolladas, para seleccionar las más eficaces y coste efectivas.

En el marco de la Estrategia NAOS se han desarrollado campañas públicas de información y educación, incluida una página web específica (http://www.naos.aesan.msps.es/); un programa escolar (PERSEO) con participación de las familias y los centros de salud con evaluación de resultados que incluye mediciones antropomórficas; convenios con la industria alimen taria para reducir grasas, azúcares y sal; puesta en marcha de un código de conducta para la publicidad de alimentos dirigidos a niños (Código PAOS) y se han establecido los Premios Estrategia NAOS. También hay que recordar que la cartera de servicios del Sistema Nacional de Salud ya incluye la prevención y el tratamiento de la obesidad entre sus prestaciones ${ }^{5}$

La Estrategia NAOS ha tenido, como no podía por menos de suceder, reflejo y desarrollo en las Comunidades Autónomas, que deben ser, dado su papel constitucional, tan protagonistas o más que el Gobierno de la Nación en la lucha contra la obesidad y los trastornos de la conducta alimentaria. Casi todas ellas tienen programas en marcha, algunos de impecable corte técnico y gran efectividad.

La obesidad, el sobrepeso y los trastornos de la conducta alimentaria constituyen, pues, un importante reto de nuestro tiempo al que los poderes públicos a todos los niveles, los profesionales y los investigadores y muchas organizaciones sociales están empezando a dar respuesta. Es un reto de tal importancia que requiere la colaboración y los esfuerzos redo blados de todos.

\section{Bibliografía}

1. Pamies Aubalat L, Quiles Marcos Y, Bernabé Castaño M. Conductas alimentarias de riesgo en una muestra de 2. 142 adolescentes. Med Clin (Barc). 2011;136: 139-143.

2. Organización Mundial de la Salud. Estrategia Mundial sobre Régimen Alimentario, Actividad Física y Salud. Ginebra, 2004. Disponible en: 
http://www.who.int/dietphysicalactivity/strategy/eb11344/strategy_ spanish_web.pdf

3. Comisión Europea. Libro blanco de la Comisión dirigido al Parlamento Europeo, el Consejo, el Consejo Económico y Social y el Comité de las Regiones. Una estrategia europea sobre las cuestiones de salud pública relacionadas con el sobrepeso y la obesidad (com(2007) XXX final. SEC(2007) aaa, SEC(2007) bbb). Bruselas, 2007. Disponible en: http://eurlex.europa.eu/LexUriServ/LexUriServ. do?uri=COM:2007:0279:FIN:ES:PDF
4. Ministerio de Sanidad y Consumo. Estrategia NAOS. Estrategia para la nutrición, actividad física y prevención de la obesidad. Madrid, 2005. Disponible en: http:// www.aesan.msps.es/AESAN/docs/docs/publicaciones_estudios/nutricion/ maqueta_NAOS1.pdf

5. Real Decreto $1030 / 2006$, de 15 de septiembre, por el que se establece la cartera de servicios comunes del Sistema Nacional de Salud y el procedimiento para su actualización. BOE 16 septiembre 2006. 